Lingüística

Vol. 33-2, diciembre 2017: 69-95

ISSN 2079-312X en línea

DOI: $10.5935 / 2079-312 X .20170018$

\title{
SINTAXIS PRONOMINAL EN LAS LENGUAS MAWETÍ-GUARANÍ: ORACIONES AGENTIVAS Y ORACIONES EXISTENCIALES
}

\author{
PRONOMINAL SYNTAX IN THE MAWETÍ-GUARANÍ LANGUAGES: \\ ACTIVE VERBAL CLAUSES VERSUS EXISTENTIAL CLAUSES
}

\author{
Wolf Dietrich \\ Universidad de Münster, Alemania \\ dietriw@uni-muenster.de
}

\section{Resumen}

Se describe la sintaxis pronominal según la jerarquía de las personas que existe en las lenguas de la gran familia Mawetí-Guaraní. ${ }^{1}$ El enfoque nuevo resulta de la interpretación de las formas mismas que se usan en la sintaxis pronominal: Cuando se trata de 1,2,3 > 3 observamos la sintaxis normal, es decir que el verbo transitivo recibe el prefijo personal de los verbos activos y el objeto pronominal aparece después del verbo si es un objeto animado; el objeto pronominal inanimado se omite. Sin embargo, cuando se trata de $3>$ $1,2 \circ 2>1$, la sintaxis cambia radicalmente, omitiéndose la marca personal sujeto del verbo transitivo.

Lo que en inglés es el pronombre de objeto (por ejemplo, en "she saw me" o "you called me"), aparece en Mawetí-Guaraní delante del verbo, en forma de pronombre personal (o de marca personal/posesiva de los nombres). Yo interpreto esta mudanza como un cambio de verbo activo con objeto a una construcción existencial: en lugar de decir "ella me vio", la mayoría de las lenguas mawetí-guaraníes dice "hubo vista mía por parte de ella". En mbyá, por ejemplo:

a'e xer-exa

3.PRON 1SG.N REL-ver/vista

Este ejemplo es especialmente significativo porque contiene la construcción típicamente nominal con el elemento REL de la flexión relacional tan característica de estas lenguas. Debido a la distinción difusa entre radicales verbales y radicales nominales en mawetí-guaraní, el cambio ("switching") de una clase de palabras a la otra es fácil.

\footnotetext{
${ }^{1}$ La familia Mawetí-Guaraní se compone de tres de las subfamilias tradicionales del tronco Tupí: la del (Sateré)- Mawé, del Awetí y la Tupí-Guaraní. Se justifica agrupar las tres familias en una gran subfamilia Mawetí-Guaraní porque ella tiene en común muchos rasgos morfosintácticos frente a las demás familias Tupí como la Arikém, Juruna, Mondé, Mundurukú, Ramarama y Tuparí.
} 
Palabras-clave: familia Mawetí-Guaraní, pronombres de objeto, diátesis activa, oraciones existenciales, cambio de las clases de palabras

In this contribution I analyze, in the languages of the South-American TupiGuarani family, the syntax of what are object pronouns in English or Spanish. The description of word classes in Tupi-Guarani languages with its fuzzy distinction between nouns and verbs is the basis of the word class switching we observe in Tupi-Guarani pronominal syntax. The existing hierarchy $1>2>$ 3 is shown by active syntax in the case of $1,2,3>3$ and $1>2$. Inanimate $3 p$ objects are included in the active verb forms and are not expressed by a special object pronoun. However, if the personal objects are higher than the subject ( $3>1,2$ or $2>1$ ), we observe a switching from active syntax with verbal person marking for subject function to nominal existential constructions. There is no more any active verbal form marked by a verbal person marker, but a nominal predicative construction characterized by nominal determination devices.

What is an object pronoun in English ("she saw me", "you called me") appears as a Tupi-Guarani personal pronoun which determines the noun-verb ("possessive" construction, such as "my seeing" = "seeing me"). Instead of saying "she saw me" speakers of most Tupi-Guarani languages say "there was her seeing me". Many basic "verbs" behave as nouns whenusing relative inflection. For instance in Mbyá:

$$
\begin{array}{lll}
a^{\prime} e & x e & r \text {-exa } \\
\text { 3.PRON } & \text { 1SG.N } & \text { REL- seeing, usually translated by 'he/she saw me'. }
\end{array}
$$

The translation, however, being pragmatically correct, does not allow understanding the structural meaning of the Mbyá expression.

Keywords: Mawetí-Guarani family, object pronouns, active voice, existential phrases, word class switching

Recibido: $28 / 10 / 2016$

Aceptado: 03/12/2016

\section{Introducción}

El objetivo de este artículo es la sintaxis de los pronombres de objeto en las lenguas Tupí-Guaraní, en mawé y en awetí (Mawetí-Guaraní). Para un número reducido de lenguas y con un enfoque metodológico diferente parte de la temática ya fue tratada en Monserrat y Soares (1983). Ese artículo, sin embargo, se limitaba a la problemática de la relación $1.5>2.0$, mientras que en el presente artículo la perspectiva de la sintaxis pronominal es más amplia.

El enfoque de este artículo está en la metodología del funcionalismo integral de Eugenio Coseriu (1981). Este funcionalismo se destaca por su "realismo", por "descubrir" (Coseriu 1981: 187) las funciones a partir de las formas y construcciones dadas en una lengua, en vez de partir de categorías 
consideradas "universales". En el caso de la sintaxis pronominal MawetíGuaraní se trata de preguntarse si hay verbos activos, transitivos, y pronombres de objecto directo en todos los casos, como se suelen describir en casi todas las gramáticas de las lenguas que nos interesan aquí, o si ciertos fenómenos sintácticos no nos inducen a buscar otros modos de análisis, a descubrir otras funciones, nunca antes descritas en los contextos sintácticos estudiados.

En cierta medida, la lingüística descriptiva actual, que muchas veces quiere ser "tipológica", comparando ciertos fenómenos a través de un gran número de las lenguas del mundo, no se ha emancipado todavía de lo que se criticaba, por ejemplo, en las gramáticas de los misioneros de la época colonial, es decir, seguir las pautas de la gramática latina.

Se continúa hablando de "genitivo" donde no hay ningún caso que se pueda comparar a un genitivo, en vez de hablar de determinación nominal o de atributos nominales. Se describen las "oraciones relativas" en una determinada lengua aunque en ella no haya ni "oración" subordinada en este caso, ni pronombre relativo, sino, por ejemplo, construcciones nominalizadas que forman atributos de otros nombres. Muchas veces las categorías que se usan son demasiado específicas para comprender las funciones genéricas de las cuales se está hablando.

La lingüística actual prefiere partir de las categorías de las lenguas conocidas, como el inglés, para aplicarlas a todas las demás, como antes se hacía con el latín. Claro está que no se pueden "inventar" categorías particulares para todas las lenguas del mundo. No se trata de esto. Pero sí se podría partir de categorías genéricas, menos idiosincráticas, distinguiendo, para retomar los ejemplos mencionados, entre diferentes tipos de atributos.

Otro ejemplo de esta actitud se verá precisamente en el tratamiento de los pronombres de objeto en verbos transitivos (cf. abajo, sección 3.). En casi todas las descripciones morfosintácticas de lenguas Mawetí-Guaraní se presentan como tales porque se piensa que se trata de sintaxis verbal activa en todos los casos en los que hay pronombres de objeto en inglés o en castellano.

En el caso de la sintaxis pronominal, el objetivo de este artículo es mostrar que la sintaxis depende de la jerarquía de las personas en las lenguas Mawetí-Guaraní. La jerarquía $1>2>3$ hace que la sintaxis cambie cuando pasamos de funciones directas (en que las personas más altas de la jerarquía interactúan con las más bajas: $1,2,3>3$ ) a funcionas agentivas inversas (cuando las personas más bajas interactúan con las más altas: $3>1,202>$ 1). Los hablantes de la mayoría de las lenguas Mawetí-Guaraní pasan entonces de la sintaxis activa a una sintaxis de construcciones existenciales. Dicho en otras palabras, la sintaxis activa conviene cuando el sujeto está en un rango más alto que la persona objeto, pero no conviene en el caso contrario. Una persona jerárquicamente baja no puede actuar sintácticamente sobre una persona más alta. La solución del aparente conflicto está en la expresión más "sociable" de las relaciones sintácticas: en lugar de la acción de uno sobre el otro se expresa la mera ocurrencia de determinaciones nominales. 
Más de una vez se ve que el funcionalismo de Coseriu va más allá de la descripción formal; siempre se busca la función semántica expresada por las formas o construcciones.

Este artículo se basa en los datos que se encuentran en las descripciones gramaticales de las lenguas de la familia Mawetí-Guaraní. Muchas de ellas tratan la temática bajo la rúbrica de la jerarquía de las personas o de los clíticos personales, sin dudar de si realmente se trata de verbos activos y de clíticos en todos los casos. Generalmente se describe solo el uso de formas diferentes, no el contenido que varía con ellas.

Dentro de las lenguas tupí-guaraní no consideramos el aché, el cocama, el omagua y el ñe'engatú, lenguas que no tienen una descendencia ininterrumpida desde el PTG y que, por eso, no presentan los fenómenos que nos interesan aquí. En todas estas lenguas la conjugación se hace mediante pronombres de sujeto y pronombres de objeto.

\section{Las clases de palabras en las lenguas mawetí-guaraní}

Como acabamos de exponer, la jerarquía de las personas se refleja en el cambio de la sintaxis de verbo activo a la de construcciones nominales predicativas, existenciales. Como esto incluye la transformación de las clases de palabras, es oportuno presentar aquí un esbozo de las clases sintácticas de las lenguas Mawetí-Guaraní.

Se distinguen en las lenguas Mawetí-Guaraní (tupi-guaraní + mawé + awetí) dos clases léxicas, verbos y nombres. Existe además la clase no léxica de los pronombres (personales, demostrativos, interrogativos e indefinidos). No existe una clase específica de "adverbios", pero sí se observan usos adverbiales de raíces nominales. No existe tampoco una clase de "adjetivos", sino que se usan verbos y nombres para expresar cualidades (véanse al respecto Dietrich 2000 y Dietrich en prensa). En el contexto de este artículo nos interesan solo verbos y nombres.

Parece que la distinción semántica entre nombres como expresiones referentes a objetos, existentes y ficticios, seres vivos e ideas, y verbos como expresiones de actividades y estados no es muy clara en las lenguas MawetíGuaraní. Dado el carácter omnipredicativo de las raíces nominales, hasta expresiones de objetos, seres vivos y estados - como, por ejemplo 'mentiroso', 'recuerdo', 'lloroso' - pueden formar predicados y corresponder así a lo que en otras lenguas se expresa mediante verbos como 'mentir', 'recordarse', 'llorar'.

\subsection{Verbos}

La distinción fácil y evidente es la que se hace de manera morfosintáctica, es decir mediante diferentes series de marcadores de persona.

Así, los verbos se identifican por los siguientes marcadores del modo indicativo (cf. Jensen 1998, 498, table 2: Proto-Tupí-Guaraní person markers, set I) 


\begin{tabular}{|llll|}
\hline 1SG & $*_{a-}$ & 1PL.EXCL & *oro- \\
& & 1PL.INCL & $*$ ja- \\
2SG & $*_{\text {ere- }}$ & 2PL & $*$ pe- \\
3 & $*_{o-}$ & & \\
\hline
\end{tabular}

Tabla 1: Marcas personales del verbo en proto-tupí-guaraní (PTG)

En toda la familia Mawetí-Guaraní se distingue una primera persona plural exclusiva, excluyendo a la $2^{a}$ persona, y una primera persona plural inclusiva. Sin embargo, se diferencia el tupí-guaraní del mawé porque este distingue entre la tercera persona del singular y del plural, mientras que el tupí-guaraní y el awetí no distinguen el número en la $3^{a}$ persona. Las marcas personales del verbo mawé y awetí son las siguientes (cf. Rodrigues/Dietrich 1997: 287-288; Borella 2000: 124; Monserrat 2012: 16-19). Para el awetí, las marcas que se ubican delante de la barra se usan con verbos intransitivos, las que están detrás de esta con transitivos:

\begin{tabular}{|c|c|c|c|c|}
\hline $\begin{array}{l}\text { Mawé } \\
\text { 1SG }\end{array}$ & $a-$ & $\begin{array}{l}\text { 1PL.EXCL.uru- } \\
\text { 1PL.INCLWa- }\end{array}$ & $\begin{array}{l}\text { Awetí } \\
a-, a j-\end{array}$ & $\begin{array}{l}\text { azo-, ozo- } \\
\text { kaj-/ti- }\end{array}$ \\
\hline $2 S G$ & $e-$ & 2PL ewe- & $e-, e j-$ & $e ? i-/ p e j-$ \\
\hline 3SG & to-/tu- & 3PL ta?atu-/te?eru-/te?ero- & $o-/ w e j-$ & \\
\hline
\end{tabular}

Tabla 2: Marcas personales del verbo mawé y awetí

Ejemplos de la marcación de persona en verbos tupí-guaraní, mawé y awetí son:

1. mbyá o-u

3-venir

'vino'

2. kayabí a-nupã kwata'í

1SG-golpear perro

'golpeé al perro'

3. kamayurá oro-je'en

1PL.EXCL-hablar

'nosotros hablamos (vos no)'

4. mawé te?ero-ket

3PL-dormir

'duermen'

5. awetí epi-atuk

2PL-bañarse

'ustedes se bañan'.

\subsection{Nombres}


Los nombres se distinguen de los verbos por marcas personales diferentes (cf. Jensen 1998, 498, table 2: Proto-Tupí-Guaraní person markers, set II). Las marcas personales nominales se derivan de los pronombres personales:

\begin{tabular}{|llll|}
\hline 1SG & *itfe & 1PL.EXCL & *ore \\
& & 1PL.INCL & *jane \\
2SG & $*$ *ere- & 2PL. & *pe...ẽ \\
\hline
\end{tabular}

Tabla 3: Pronombres personales del PTG

No se puede reconstruir un pronombre personal de $3^{a}$ persona, ya que este se ha formado a partir de demostrativos en las diferentes lenguas TupíGuaraní. Por razones de espacio no vamos a listar aquí los pronombres personales del mawé y del awetí, pero podemos afirmar que estos también están a la base de las marcas de persona nominales (cf. Rodrigues/Dietrich 1997: 292-294; Borella 2000: 72; Monserrat 2012: 23-25). El awetí se caracteriza por la distinción entre el habla de los hombres y la de las mujeres en la $3^{a}$ persona.

\begin{tabular}{|c|c|c|c|c|}
\hline \multicolumn{3}{|c|}{ Proto-tupí-guaraní } & Mawé & Awetí \\
\hline $1 \mathrm{SG}$ & *tfe- & $\begin{array}{l}\text { 1PL.EXCL*ore- } \\
\text { 1PL.INCL. *jane- }\end{array}$ & $\begin{array}{c}u-\quad \begin{array}{c}\text { uru- } \\
a^{-}\end{array}\end{array}$ & $\begin{array}{l}\text { azo-/ozo } \\
\text { kaj- }\end{array}$ \\
\hline $2 \mathrm{sG}$ & $* n e-$ & 2PL. $\quad * p e(n)-$ & $e^{-} \quad e^{-}$ & epi- \\
\hline 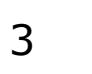 & $*_{i-/ t s-/ t}$ & & $i-/ h(e)-/ s-$ & $n(\tilde{a})-($ h.masc. $) / i-/ t-($ h.fem. $)$ \\
\hline
\end{tabular}

Tabla 4: Marcas personales nominales en Mawetí-Guaraní

Ejemplos de la marcación de persona en raíces nominales tupí-guaraní, mawé y awetí son:

6. guar. occidental che-mémby

'mi hijo/hija' (habla de la mujer)

7. guar. occidental ché che-mémby

1SG.PRON 1SG-hijo/hija

'hay mi(s) hijo(s)/hija(s)', 'tengo niños'

8. guar. occidental o-pá-ete kía-réta o-mbotávy-va agwára ij-ápu

3-estar.completo-INT alguien-PL 3-engañar-NOR zorro 3-mentira

'las mentiras del zorro son tales que engañan a todos'

9. guar. occidental ché che-apũ-a

yo 1 SG-mentira-NEG

'yo no miento', 'digo la verdad'

10. guarayu i-maendua ndé-retse

3-acordarse 2SG.PRON-LOC

'se acuerda de ti', 'te manda saludos'

11. wayãpi e-aĝywo (Copin inédita: 230)

1SG-tristeza/triste

'mi tristeza'/ 'estoy triste' 
12. wayãpi

13. mawé

14. awetí e-memy ije

1SG-hijo 1SG.PRON

'tengo un hijo/hija', 'tengo hijos (mujer hablando'

u-h-eha

'mi(s) ojo(s)

u-he-sirat

'mi hambre'/'tengo hambre'

i-mẽpýt akój

3-hijo aquel

'aquel es mi hijo (mujer hablando'.

Los ejemplos (7), (9) y (12) muestran que, con sujetos pronominales, la predicatividad de las oraciones existenciales generalmente se produce mediante la exposición del pronombre personal junto con el nombre marcado por el morfema de la persona.

\subsection{La flexión relacional y el problema de la distinción entre verbos y nombres}

Todas las lenguas tupí-guaraní que presenten una descendencia ininterrumpida desde el proto-tupí-guaraní y que no hayan reorganizado posteriormente partes de su morfosintaxis han gramaticalizado prefijos que marcan determinadas relaciones sintácticas. ${ }^{2}$ En una perspectiva sincrónica, se trata de raíces léxicas básicas con inicial vocálica las que reciben determinados prefijos consonánticos, según su función sintáctica. Generalmente, hay tres posibilidades:

- el antiguo */ts/-, que aparece, según la evolución fonológica, como ts-, $s^{-}$, $h$ - $0 \varnothing$-, tiene la función de la marca de la $3^{a}$ persona, posesiva 0 predicativa, y así se comporta como un alomorfo de $i$-: tupinambá s-asy 'su dolor', 'le duele', 'está enfermo' $\approx$ guaraní paraguayo $h$-asy; asuriní del Tocantins, uru-eu-uau-uau (kagwahib) h-ahy, mbyá ø-axy. No funciona en lenguas como el aché, el siriono o kokama. En esta última lengua se ha lexicalizado en lexemas como tsáku 'calor, caliente' <*tsaku, tsachi 'dolor' <*ts-atfy, yakitsa 'cabello' <yaki 'cabeza' + tsa 'cabello' $<$ PTG *ts-a $\beta$.

- el resultado $r$ - expresa la contigüidad entre un atributo (determinante) y el nombre determinado que le sigue. El nombre marcado por $r$ - se vuelve así determinado. Esta contigüidad se da no solo entre determinantes y determinados nominales, sino también entre nombres determinados por

\footnotetext{
2 Meira y Drude (2013) desarrollaron la hipótesis esbozada ya en Rodrigues y Dietrich (1997: 289-290) según la cual una */t/- del proto-mawe-guaraní habría evolucionado a $* / t /, * / t /$ y $* / r /$ en diferentes posiciones. Los tres resultados diferentes se habrían gramaticalizado en las lenguas tupí-guaraní para establecer la llamada flexión relacional. En mawé se han conservado restos de $* / \mathrm{ts} /->/ \mathrm{h} /$ - en la función de la $3^{a} \mathrm{p}$, predicativa o no, mientras que observamos una reorganización diferente para el resultado /r/-. En awetí no hay ningún prefijo relacional funcional, a pesar de poder aparecer un $-t-$, eufónico, intercalado entre dos vocales (Borella 2000: 69-70).
} 
pronombres personales de $1^{a}$ y $2^{a}$ persona, los cuales así revelan el carácter más bien pronominal de los determinantes posesivos o marcas personales nominales:

15. tupinambá, guaraní paraguayo xe $r$-u $r$-asy

yo REL-padre REL-dolor

'el dolor/la enfermedad de mi padre'

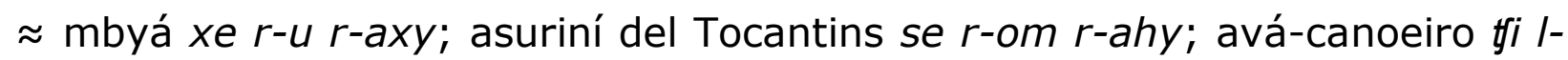
$u$ I-ai. En siriono, $r$ - se ha lexicalizado en lexemas cognados, por ejemplo en siriono ra 'cabello' < PTG*a $\beta$, siriono raku 'caliente' < PTG *aku $\beta$, siriono rae 'camino' < PTG *ape.

- en tercer lugar, los resultados $t$-, a veces $s \circ \emptyset$, indican la ausencia de cualquier función sintáctica. Esto se da cuando no hay ninguna determinación atributiva (uso genérico). Ejemplos son: guaraní paraguayo t-a'y 'hijo (varón hablando)', t-embireko 'esposa', pero s-o'o 'carne', okẽ 'puerta' (morfológicamente ø-okẽ, óga r-okẽ 'la puerta de la casa'); warázu/guarasu $t$-ə̇ 'diente', t-ié 'barriga', $t$-o?o 'carne'; wayãpi t-ea 'ojo(s), t-etymã 'pierna', $t$-ãj 'diente(s); parakanã $t$-ow 'padre', $t$-iró 'recipiente', ø-aワ 'casa'. La función no existe ni en mawé ni en awetí.

En lo siguiente (cf. 3.) no veremos solo el cambio de una clase de palabras a la otra, sino también la apariencia del morfema relacional $r$ - $/$ - en construcciones con verbos "nominalizados", es decir con verbos que se comportan como nombres. Véanse los ejemplos 25. ndee xe-r-apy 'vos me quemaste', de la siguiente sección del mbyá; 40. Tumpã nde r-aytsu 'Dios te ama', de la del guarayu; 86. tfé r-enom ipé 'vos me oís', de la del parakanã; 108. ure-r-etfa ku 'él nos vio', de la del araweté; 144. na-je-r-esag-i-'gã 'él no me vio', de la del kayabí; 192. u-h-enoi '(ellos) me enseñan', de la del mawé. En estos ejemplos tenemos que ver con raíces que no se comportan como verbos transitivos, con sus marcas personales verbales de sujeto $y$, dado el caso, con objetos nominales o pronominales jerárquicamente inferiores a la persona del sujeto, sino que observamos las mismas raíces desprovistas de sus marcas personales de sujeto, caracterizadas por el morfema relacional de contigüidad que indica la determinación por un pronombre. Se trata, pues, de construcciones nominales, a base de verbos que han cambiado a la clase nominal.

En lo que sigue se describirá cómo funciona la sintaxis pronominal de las lenguas Mawetí-Guaraní.

\section{La sintaxis verbal agentiva contra las oraciones existenciales nominales}

En la mayoría de las lenguas mawetí-guaraní observamos una jerarquía personal $1>2>3$. Esto quiere decir que el hablante es jerárquicamente más alto que el interlocutor y que ambos son más altos que las personas que no participan en el discurso. Las terceras personas que se refieren a objetos 
inanimados (cosas) ocupan el puesto más bajo en esta jerarquía. Por esto, muchas veces, no pueden figurar como sujetos, ni se expresan como complementos de objeto.

De esta jerarquía personal resulta una diferenciación sintáctica:

1) En construcciones transitivas, las personas interlocutorias tienen el papel de sujeto cuando el complemento de objeto directo es jerárquicamente inferior $(1>2,3 ; 2>3)$. Hay construcción activa, con formas verbales agentivas, también en el caso de la igualdad de dos o más terceras personas no idénticas $(3>3)$. En el caso de $1>2$, tanto en el singular como en el plural, hay marcas personales específicas en muchas lenguas mawetí-guaraní tradicionales, llamados morfemas portemanteau por algunos lingüistas (PTG *oro-, *opo-).

2) Una persona jerárquicamente inferior no puede "actuar sobre" una persona más alta $(2>1 ; 3>1,2$ en lenguas europeas como el español o el inglés). En este caso, la transitividad verbal es imposible. Observamos el pasaje (switching) de una construcción verbal activa a una construcción existencial, nominal, inactiva del tipo 'hay/hubo acción $x$ mía/tuya/nuestra/vuestra (a partir) de él/ella/ellos/ellas' o 'hay/hubo acción x mía/nuestra (a partir) de ti/vosotros'. El indicio de la construcción nominal es la falta de la marca personal activa del "verbo" (que pasa a volverse nombre) y, en los verbos de la clase $h-/ r$ - inicial la marca $r$ - de la flexión relacional, típica de los elementos nominales.

En realidad, se trata de una predicación nominal con determinación doble: Mbyá [a'e $\rightarrow$ [xe $\rightarrow$ r-exa]] ['hay vista mía'] o [de éln $\rightarrow$ ['hay ver $\left.\rightarrow \mathrm{me}^{\prime}\right]$ ] o 'hay su verme'

Kayabí [waiwa kyna $\rightarrow$ [je $\rightarrow$ mu'jaù]] ['de la mujer vieja $\rightarrow$ [hubo enseñar $\rightarrow$ me]].

Se ve que no basta decir que en tal o cual otra lengua hay una jerarquía de las personas gramaticales, sino que hay que explicar lo que significa esta jerarquía. La lengua no es un mero conjunto de formas agrupadas según ciertas reglas, sino que es un todo significante. Un cambio de construcción implica otro aspecto de la descripción lingüística del mundo, otros significados. En esta situación, no basta decir que en tal caso se usan otras marcas personales, sino que hay que justificar el cambio funcional que implican las marcas personales agentivas del verbo, por un lado, y construcciones nominales, no agentivas, por el otro.

\subsection{Documentación en las lenguas mawetí-guaraní}

A continuación listamos ejemplos de oraciones existenciales nominales en todas las lenguas suficientemente descritas que las presenten. Será solo excepcionalmente que damos también ejemplos de construcciones verbales agentivas, bien conocidas. Después de las explicaciones morfológicas y morfosintácticas dadas en la sección 2. y por razones de espacio, renunciamos a glosas en la mayoría de los casos. Las ofrecemos cuando nos parecen 
indispensables. Varias lenguas se caracterizan por exponer un pronombre de $2^{a} \mathrm{p}, \mathrm{sg}$ y $\mathrm{pl}$, al final del sintagma para marcar la relación $2>1$ (guarayu, warazu, asuriní del Tocantins, parakanã, tapirapé, asuriní del Xingú, kayabí, wayãpi, emerillon).

Guaraní paraguayo (Guasch 1956: 97-99)

16. ha'e che r-echa

3.PRON 1SG.N REL-ver

'él/ella me ve/vio'

17. ha'e nde r-a'arõ

'él/ella te espera/esperaba'

18. nde che mbotavy

'vos me engañaste'

19. ha'e nde nupã-ta

3.PRON 2SG.N golpear-FUT

'él/ella te golpeará

20. pende che re-rahá-ta Paraguaý-pe

2PL 1SG.N REL-llevar-FUT Asunción-LOC

'ustedes me llevarán a Asunción'

Mbyá (Dooley 2006, ss. vv.mbo'e, -exa, -apy)

21. n-ore-mbo'é-i

NEG-1PL.EXCL.N-enseñar-NEG

'no nos enseñó'

22. t-ore-mbo'e!

HORT-1PL.EXCL-enseñar

'ique nos enseñe!'

23. ava xe-mbo'e

'el hombre me enseñó'

24. a'e xe-r-exa

'él me vio'

25. ndee xe-r-apy

2.SG.PRON 1SG.N-REL-quemar

'vos me quemaste'

26. João xe-r-exa kuee

João 1SG.N-REL-ver ayer

'João me vio ayer'

Guaraní Occidental (Chiriguano) (Dietrich 1986: 158-161)

27. háe che-r-écha

3SG.PRON 1SG-REL-ver

'él me vio'

28. nde che-nũpa

2SG.PRON 1SG.N-castigar

'vos me castigás' 
29. pénde ore-r-ẽnei

2PL.PRON 1PL.EXCL-REL-Ilamar

'ustedes nos llamaron'

30. háe ne-nõe

3SG.PRON 2.SG.N-sacar

'ella te sacó'

Tapiete (González 2005: 148-154)

La sintaxis de las demás lenguas mawetí funciona solo parcialmente en tapiete. Los dos primeros ejemplos muestran que en tapiete la construcción verbal ni-mingiye 'vos me asustaste' puede confundirse con la nominal shimingiye, confusión que observamos también en el último ejemplo. Mientras que el morfema iri' '1 $>2$ ' se usa en el primero de los dos últimos ejemplos, no aparece en el anterior. Este presenta una construcción existencial nominal, no usual en otras lenguas en el caso de $1>2$.

31. nde shi-mingiye

2SG.PRON 1SG.N-asustar

'vos me asustaste'

o también

32. nde ni-shi-mingiye

2SG.PRON 2SG-1SG.N-asustar

'vos me asustaste'

33. ndi-wohi-ha

2sG.N-Ilevar-1PL.EXCL

'te llevamos'

34. iri-r-esha

$1>2$-REL-ver

'te veo'

variante de:

35. a-ri-r-esha

1SG.S-2SG.O-REL-ver

'yo te veo'

Guarayu (Hoeller 1932: 83-88)

36. che oro-kuaa

1SG.PRON 1SG.S > 2sG.o-conocer

'yo te conozco'

37. opo-kuaa

'os conozco'

38. che-kuaa ebe

1SG.N-conocer vos

'vos me conocés' 
39. ore-juka peje!

1PL.EXCL.N-matar 2PL.S > 1PL.EXCL.O

¡Mátennos!

40. Tumpã nde r-aytsu

'Dios te ama'

41.a'e che-kuaa

'él/ella me conoce'

42. aipo che r-epy

'él me vengó'

43. nde kuri nde mbo'e-ra porombo'etsar

2.SG.PRON después 2.SG.N enseñar-FUT profesor

'a vos te enseñará después el profesor'

Warázu (tradicionalmente guarasu o pauserna; Ramirez \& de França, 2017)

44. oro-núpä

'yo te castigué/nosotros te castigamos'

45. uru-épa

'te vi/te vimos'

46. ai-núpä

'lo castigué'

47. a-h-épa

'lo vi'

48. tse-núpä

'me castigó'

49. tse-r-épa

'me vio'

50. zane-núpä

'nos (incl.) castigó'

51. zane-r-épa

1PL.INCL-N-REL-ver

'hubo vernos (por parte de él)', 'nos (incl.) vio'

52. tse-núpä ápe

1SG.N-castigar 2SG.S > 1SG.O

'vos me castigás'

53. tse-r-épa ápe

'vos me viste'

54. tse-núpä pezópe

1SG.N-castigar 2PL.S > 1SG.O

'ustedes me castigaron'

55. tse-r-épa

'ustedes me vieron'

Yuki (Villafañe 2004: 107-108; 209) 
56. are-yukia

1SG.S > 2SG.O-matar

'yo te mato'

57. ha-yukia

'yo os mato'

58. ua che-yukia

'él me mata'

59. ua de-yukia

'él te mata'

60. chi-orõ

'me sujeta'

61. ua ch-icha

'él me ve'

Siriono (Schermair 1949: 330-340; Priest y Priest 1985: 33)

62. are-gatobi

'yo te quiero'

63. are-noã ha

'yo os castigo' (Priest y Priest 1985: are-iruãnua)

64. (nde) se rea

'vos me ves'

65. nde gatobi se $=$ se nde gatobi

'vos me querés'

66. nde ure noã = ure noã nde

'vos nos castigás' (Priest y Priest 1985: nde ure nua; ure iruã nua nde)

67. (ae) se noã

'él me castiga' (Priest y Priest 1985: se iruã nua)

68. (ae) se rea

'él me vio'

69. (ae) nde noã

'él te castiga'

Tupinambá (Anchieta 1595: 11-12, 38)

70. xe-r-epják jepé

1SG.N REL-ver 2SG.N

'vos me viste'

71. xe-r-epják jepejé

'ustedes me vieron'

Avá-Canoeiro (Borges 2006: 158-161)

72. tyi-tõ ni-tõ a-kutuk

1SG.PRON-PART 2SG.PRON-PART 1sG-taladrar

'yo te taladré' 
73. jane-nupã

'(ellos) nos pegaron'

74. wira na-potok

'el árbol te pilló'

75. ore-moe

'(ella) nos enseñó'

76. ni-tõ tij-karaj

'vos me rasguñaste'

Asuriní delTocantins (Cabral/Rodrigues 2003: 226)

77. oro-soka-pota

$1>2$-matar-querer

'voy a matarte'/'vamos a matarte'

78. a-soka-pota

1SG-matar-querer

'voy a matarlo'

79. o-se-soka pota

'él va a matarme'

80. ore-soka-potar ipe

1PL.EXCL-matar-querer 2.N.S>1.N.O

'vos vas a matarnos/ustedes van a matarnos'

81. pehé isé-soká-okár ipé

2PL.PRON 1SG.N.O-matar-FACT 2PL.AGENTE

'hizo que ustedes me mataran'

82. oré-nopo ipé

'ustedes nos castigaron'

Parakanã (Souza e Silva 1999: 53; 71-72)

83. oro-tfoká

1.s>2sG.o-matar

'yo te mato/nosotros (EXCL.) te matamos'

84. itfé a-itfán áwa

1SG.PRON 1SG-ver gente

'yo veo a ustedes'

85. ené tfé r-ewimamán ipê

'vos me empujás'

86. tfé r-enom ipé

1SG.N REL-Oír 2SG.ERG

'hay oírme por parte de vos', 'vos me oís'

Souza e Silva (1999: 53) tiene buena intuición de la sintaxis cuando escribe que ipé es un pronombre ergativo de $2^{a} p$ que actúa sobre una $1^{a}$ persona. Ergatividad significa que no hay verbalidad activa, no hay ni sujeto ni objeto. En nuestra interpretación no se trata de ergatividad, sino de 
construcciones existenciales nominales. En el ejemplo (85) del parakanã analizamos tfé r-ewimamán '(hay) empuje con respecto a mí o 'hay empujarme', y este sintagma está determinado por ené 'vos': 'ocurre empujarme por parte de vos', 'vos me empujás'. El pronombre ipé 'vos' es un focalizador que marca el tema del enunciado, que es la $2^{a}$ persona. La relación $1 . S>2 \mathrm{PL}$. se expresa mediante el verbo y el objeto áwa 'gente'.

Suruí-Aikewara (Lopes 2014: 136-138)

87. ise puta uru-nupo

1SG.PRON querer 1SG.S > 2sG.O-golpear

'yo voy a golpearte'

88. ene puta ti-nupo pe

2SG.N querer 1SG.N-golpear 2SG.ERG

'vos vas a golpearme'.

Para nuestra interpretación del "ergativo" véase la sección anterior del parakanã.

89. ajkwes-a ti-asuron

aquel-ARG 1SG.N-abrazar

'aquel me abrazó'

Tembé-Tenetehara (Duarte 2007: 46)

90. dane-duka-rəm dawar

'nos matará el tigre'

91. ne-duka-rəm dawar

'el tigre te matará'

92. ne he-r-aro-rəm

'vos me esperarás'

Tapirapé (Praça 2007: 103-105)

93. ãxe'i rãka a-a xe-r-exãk-a

ayer PAS.REC 3-ir 1S-REL-ver-GER

'ayer fue a verme'

94. ã-ã rãka ne-r-exãk-ire

1SG-ir PAS.REC 2SG-REL-ver-ANT

'fui después que te vi'

95. pãxe xe-pyter

payé 1SG.N-soplar

'el payé me sopló'

96. xe-ma'ẽ xepe

1SG.N-enseñar 2SG.S >1SG.O

'vos me enseñás'

97. peẽ xe-maky'ã pexepe

2PL.PRON 1SG.N-ensuciar 2PL.S>1SG.O

'ustedes me ensuciaron' 
98. peẽ are-r-exãk arepe

2PL.PRON 1PL.EXCL.N-REL-ver 2PL.S>1PL.EXCL.O

'ustedes nos miraron'

99. xe-ãrõ

'ellos me esperan'

100. ã-ãrõ

'yo le(s) espero'

101. ne-ãrõ

'ellos te esperan'

102. xe-ãrõ xepe

'hay esperarme por parte de vos', 'vos me esperás'

103. a-ãrõ

'ellos los esperan'

104. ere-ãrõ

'vos lo(s) esperás'

105. are-ãrõ arepe

'hay esperarnos por parte de vos', 'vos nos esperás',

pero, en el caso de 1.S > 2.0:

106. ara-pyro

1SG.S > 2SG.o-ayudar

'yo te ayudo'

107. ãpa-nopỹ

1SG.S > 2PL.O-golpear

'yo os golpeo'.

Araweté (Solano 2009: 352-358)

108. ure-r-etja ku

'él nos vio' ( $k u$ FOC)

109. ne-r-etfa ku

'él te vio'

110. he-r-etfa ku ne

1SG.N-REL-ver FOC 2SG.N

'vos me viste'

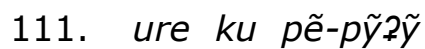

1PL.EXCL.PRON FOC 2PL.N-pellizcar

'pellizcamos a ustedes'

112. he ku ne-nupĩ

'(yo) te pegué'

113. pẽ-nupĩ ku he

'yo pegué a ustedes' (la construcción con un prefijo portemanteau no existe).

Asuriní del Xingú (Pereira 2009: 122-124; 243-247) 
114. dje ga a-djuka

1SG.PRON 3SG.PRON 1SG-matar

'yo lo maté'

115. ure uru-puru-munik

1PL.EXCL.PRON 1PL.EXCL-GEN-empujar

'nosotros empujamos a la gente'

116. uru-etxak

1SG > 2SG-ver

'yo te vi'.

Pero, en construcciones nominales:

117. dje r-etxak

1SG.N REL-ver

'hubo verme', 'él me vio'

118. dje $p y^{\prime} y k$

1SG.N agarrar

'hubo agarrarme', '(él/ella) me agarró'

119. dje r-etyk ape

1SG.N REL-derrumbar 2SG.S >1SG.O

'vos me derrumbaste'

120. pene ure $p y^{\prime} y k$ pedjepe

2PL.PRON 1PL.EXCL.N agarrar 2PL.S>1PL.EXCL.O

'ustedes nos agarraron'.

Anambé (Julião Inédita: 73-75)

121. ne-hy jene-r-ekã

'tu madre nos espera'

122. na jene-r-ekã

'vos nos esperás'

123. uru-ne-r-ekã xa

1PL.EXCL.N-2SG.N-REL-encontrar NEG

'no te hemos encontrado'

124. kurumĩ ne-r-ekã

'el muchacho te espera'

125. jawãtĩ uru-u'u

'el perro nos mordió'

126. $\tilde{u}-i h a-a j \tilde{y}$

3.N-1SG.N-empujar

'él me empuja'

127. iha-ũ-ajỹ

1SG.N-3.N-empujar

'hay su empujarme', 'yo lo empujo'. 
Los dos últimos ejemplos muestran que en anambé las construcciones nominales no se hacen con los pronombres independientes ( $j a$ 'yo', wy 'él, ella), sino exclusivamente con los "clíticos", las marcas personales nominales.

Kayabí (Dobson 1997: 53-54; Gomes 2007: 20-25; Braga 2016: 29-32)

128. a-nupã je 'gãa

1SG-golpear 1SG.PRON.S 3SG.PRON.O

'lo golpeé'

129. a-nupã je ene

'te golpeé'

130. oro-nupã ore kĩã

'lo golpeamos (mujer hablando)'

131. ere-nupã 'gã

'vos lo golpeás'

132. pe-nupã 'gã

'ustedes lo golpean'

133. si-nupã jane kĩã

'nosotras lo golpeamos'

134. o-nupã 'gã 'gã

'él lo golpea'

Estos primeros ejemplos muestran que en el caso de $1,2,3>3$ y de $1>$ 2 se usan verbos activos. El complemento de objeto pronominal está siempre en la última posición, detrás de eventuales pronombres de sujeto. En los casos de $2>1$ y de $3>1$, 2 observamos construcciones existenciales nominales, con los pronombres ape '2SG.S > 1SG.O' pejepe '2PL.S > 1PL. EXCL.O' en la última posición, como todos los pronombres de sujeto focalizados.

135. je-nupã ape

1SG.Ngolpear 2SG.S > 1SG.O

'vos me golpeás'

136. ore-nupã ape

'vos nos golpeás'

137. ore mono ape

'vos nos mandás'

138. ore-nupã pejepe

'ustedes nos golpean'

139. je-nupã 'gã

'él me golpea'

140. je monoo-nupã 'gã

'él me manda'

141. ene-nupã 'gã

'él te golpea' 
142. waiw-a kyna je-mu'j-aù t-aity are mujer.vieja-ARG 3sg.FEM.PRON 1SG.N-enseñar-NARR REL-hamaca sobre 'una mujer vieja me enseñó hacer hamacas' o 'hubo enseñarme de la mujer vieja'

143. je mo-py'apojy ty'ar-a 1SG.N CAUS-vomitar hambre-ARG 'el hambre me hizo vomitar'

144. na-je-r-esag-i 'gã NEG-1SG.N-REL-ver-NEG él 'él no me vio'

145. jasi'y-a n-ore- mõ-ger-uka-i mosquito-ARG NEG-1PL.EXCL.N-CAUS-dormir-FACT-NEG 'el mosquito no nos deja dormir'

146. 'gã neeraw-au je

él Ilevar-NARR 1SG.N 'yo lo llevé'

Kagwahib (Betts 2012, ss. vv. -epiag, nupã, pihãpihã, u'u)

147. o-ma'e-gãtu ga h-epiag-a (Uru)

3-despertarse-bien 3.PRON 3-ver-GER

'Se despertó bien y lo vio' ('ocurrió su vista por parte de él [de otro]')

148. a'ero ga nda-ji-r-epiag-i (Uru)

entonces 3SG.PRON NEG-1SG.N-REL-ver-NEG

'entonces no hubo mi vista por parte de él', 'entonces no me vio'

149. ji-pihãpihã ga (Tenharim)

1SG.N-pellizcar 3SG.M.PRON

'él me pellizcó repetidamente'

150. oro-nupã

'yo te golpeo'

151. opo-nupã

'os golpeo'

152 pe-nd-epiag-a ji-ruri (Uru)

2PL-REL-ver-GER 1SG.N-venir

'Vine a verles a ustedes'

153. o-ho hẽa ga-r-epiak-a

3-ir 3SG.FEM.PRON 3SG.M.PRON-REL-ver-GER

'ella fue a verlo'

154. o-u'u ga ga

3-morder 3SG.PRON.M.O 3SG.PRON.M.S

'él lo mordió' o, mejor, 'lo mordió él'

155. ga u'u mboj-a

3-SG.PRON.M.O morder serpiente-ARG

'una serpiente lo mordió'

156. ja'gwára nda-ji-u'u-i

tigre NEG-1SG.N-morder-NEG

'el tigre no me mordió' 
En Kagwahib no se distingue el pronombre de $3^{a}$ persona objeto animado del inanimado. Hasta el inanimado se expresa después del verbo.

Wayãpi (Copin inédita: 239-243)

157. e-poãnũ

1SG.N-curar

'(él/élla) me ha curado'

158. ole-poãnũ

1PL.EXCL.N-curar

'(él/ella) nos ha curado'

159. ne-poãnũ

2sG.N-curar

'(él/élla) te curó'

pero, con verbos activos y los semi-auxiliares -jpa 'progresivo' y -kupa 'colectivo':

160. ele-poãnũ e-jpa

2SG-curar 1SG.N-AUX

'vos me curaste'

161. pe-poãnũ ole-kupa

2PL-curar 1PL.EXCL-AUX

'ustedes nos curaron'.

162. olo-poãnũ

1SG > 2SG-curar

'yo te curé'

163. o-poãnũ

3-curar-ø

'él lo curó'.

Parece que en wayãpi la jerarquía de las personas es $1,2>3$, ya que las relaciones $1>2$ y $2>1$ se expresan con verbos activos y solo las relaciones 3 $>1,2,3$ se construyen mediante oraciones existenciales nominales, de manera análoga al emerillon, cuyo comportamiento sintáctico se ve en los ejemplos siguientes:

Emerillon (Rose 2011: 72-81)

$3>1,2$

164. zawar e-su2u

'un perro me mordió'

165. zawar de-su2u

'el perro te mordió'

$2>1$ : se hace con verbos activos y con pronombres más bien opacos que siguen al verbo: 
166. ere-raho eren

'vos me llevaste'

167. pe-kuar oreren 'ustedes nos llevaron'

$1>2$ :

168. e-wedzu t-oro-pu!

2SG.IMP-bajar HORT-1sg.S > 2sg.O-comer

ibaja que yo te coma!'

169. aporo-ekar

1PL.EXCL.S > 2PL.O-buscar

'hemos buscado a ustedes'

Guajá (Magalhães 2007: 192-197)

170. nijã ha-r-ixá

2SG.PRON 1SG.N-REL-ver

'vos me viste'

171. pijã ha-r-ixá

2.PL.PRON 1SG:N REL-ver

'ustedes me vieron'

172. nijã are-r-ixá

2SG.PRON 1.PL.EXCL.N-REL-ver

'vos nos viste'

173. ha-r-ixá a'iá

1SG.N-REL-ver 3.PRON

'me vió/me vieron'

174. jahá ni-n-ixá

1SG.PRON 2SG.N-REL- ver

'yo te vi'

175. areá ni-n-ixá

1PL.EXCL.PRON 2SG.N-REL- ver

'te vimos'

176. aréa pĩ n-ixá

1PL.EXCL.PRON 2PLN REL-ver

'nosotros vimos a ustedes'.

Se ve que en guajá la jerarquía de las personas es $1,2>3$, ya que se expresa con verbos; pero, a diferencia del wayãpi y del emerillon, se han generalizado las construcciones existenciales nominales también en el caso de 1.S > 2.0. Las personas intralocutivas se consideran, pues, igualitarias, por oposición a la $3^{a}$ persona, extralocutiva.

Urubú-Ka'apor (Kakumasu 1986: 379-381)

Funciona difícilmente, ya que las marcas personales del verbo se usan regularmente solo con raíces monosilábicas. Además, la función de objeto se 
indica mediante la partícula ke, que sigue al complemento de objeto. Los morfemas portemanteau se desconocen.

Parece que la ante- o posposición del objeto no depende del carácter pronominal del mismo.

177. ihẽ nde ke a-raho-tar katu tipe

1SG.N 2SG.N O 1SG-llevar-FUT bien FRUST

'quisiera bien llevarte (pero no puedo)'

178. ø-mu-hury katu ihẽ ke ngã

3-CAUS-alegre bien 1SG.PRON OBJ 3PL.PRON

'ellos me reconfortaron bien'

179. ihẽ ke a'e mu'e tĩ

1SG.PRON OBJ 3.PRON enseñar también

'él me enseñó también'

180. ihẽ ne ke a-kutuk-ta

1SG.PRON 2SG.PRON OBJ 1SG-taladrar-FUT

'yo te taladraré'

Kamayurá (Seki 2000: 63; 137-140)

181. je r-etsak

1SG.N REL-ver

'hay vista en cuanto a mí', 'vos me ves'

182. je-tsaro-k

1SG.N-buscar-VOL

'iven a buscarme!'

183. oro-etsak

1SG.S > 2SG.O-ver

'yo te veo/nosotros te vemos'

184. opo-etsak

1SG.S > 2PL.O-ver

'yo veo/nosotros vemos a ustedes'

Mawé (Rodrigues/Dietrich 1997: 293-295)

185. a-ti-kuap

1SG-3-conocer

'lo(s)/la(s) conozco'

186. aro-kuap

1SG.S > 2SG.O-conocer

'(yo) te conozco'

187. war-enoi

1PL.EXCL.S > 2SG.O-enseñar

'te enseñamos'

188. e-ti-kuap

2SG-3-conocer

'lo(s)/la(s) conocés',

pero sin marca personal de sujeto, en construcciones nominales existenciales: 
189. u-h-enoi

1SG.N-3-enseñar

'hay su enseñanza con respecto a mí'

'ellos me enseñan'

191. a-h-enoi

1PL.INCL.N-3-enseñar

'nos enseñan (a mí y a ti)'
190. h-enoi

3-enseñar

'hay la enseñanza de ellos', 'lo enseñan', 'es enseñado'

192. uru-h-enoi

1PL.EXCL.N-3-enseñar

'hay su enseñanza para nos', 'nos (excl.) enseñan'

Awetí (Borella 2000: 136-149; Monserrat 2012: 19-25)

No existen las marcas personales portemanteau correspondientes a PTG *oro-, *opo-. Parece que la posición final del pronombre de sujeto marca foco:

193. atit a-t-apit $e n^{3}$

1SG.PRON.M 1SG-t-quemar 2SG.N

'yo te quemé'

Sin embargo, sin el prefijo de persona, en construcciones existenciales nominales:

194. it-apit en

1SG.N-quemar 2SG.PRON

'ocurrió quema de mí por parte de vos', 'vos me quemaste'

195. en azo-apit

2SG.N 1PL.EXCL-quemar

'hubo quema de nosotros por parte de vos', 'vos nos quemaste'

196. kujtã e2i-apit

DEM 2PL.N-quemar

'aquel quemó a ustedes'

197. kujta-za kaj-apit

DEM-COL 1PL.INCL.N-quemar

'ellos nos quemaron'.

\subsection{Clasificación según el funcionamiento de la jerarquía de las personas}

I) Funcionamiento de construcciones nominales inagentivas en los casos 2 $>1,3 ; 3>1$, 2. Marcas personales activas en los casos 1, 2, $3>3$. Morfemas portemanteau en los casos $1>2$ (con restricciones en siriono y parakanã, 1 > 2PL): guaraní paraguayo, mbyá, guaraní occidental, guarayu, yuki, siriono, parakanã, suruí-aikewara, tembé, kagwahib, kamayurá, mawé

II) Variante de la jerarquía de las personas: 1 y $2>3$. De ahí que $1>2$ se construya con marcas verbales portemanteau, y $2>1$ se construya

${ }^{3} \mathrm{La}$ - $t$ - en $a$-t-apit en es una /t/ eufónica (cf. nota 2 arriba). 
igualmente con verbos activos. Solo en las relaciones $3>1,2,3$ se usan construcciones existenciales nominales: wayãpi, emerillon

III) Funcionamiento parcial: los prefijos portemanteau $1>2$ no existen o no se usan obligatoriamente (tapiete); extensión de las construcciones agentivas. En el caso del anambé, observamos extensión de las construcciones nominales: tapiete, avá-canoeiro, araweté, anambé, kayabí, guajá, awetí

El kayabí se caracteriza por pronombres específicos de $2^{a} \mathrm{p}$ (ape, pejepe; véase después del ejemplo 134), por la posición final del sujeto (de los pronombres de sujeto/de referencia última en una construcción nominal con doble determinación) y por la falta de los morfemas portemanteau.

IIIa) Funcionamiento en uno de los tres grupos anteriores, pero con pronombres específicos para la $2^{a}$ persona agente: guarayu, warazu, tupinambá, asuriní del tocantins, parakanã, tapirapé, kayabí, asuriní del xingú, wayãpi, emerillon.

IV) Sin jerarquía. Construcciones verbales activas en todos los casos: urubúka'apor. El aché, kokama-omagua y ñe'engatú, si se clasifican, se clasifican aquí. Pero, como ya dijimos arriba (cf. final sección 1), estas lenguas no heredaron o no conservaron la morfosintaxis tradicional TupíGuaraní ni siquiera tienen los instrumentos para oponer construcciones agentivas y construcciones existenciales nominales.

Se destacan así tres grupos, con dos subgrupos, pues cinco áreas en las cuales la jerarquía de las personas funciona, pero se manifiesta de manera variada. En ellos observamos el cambio de la sintaxis verbal agentiva a construcciones existenciales nominales. Un cuarto grupo se caracteriza por la ausencia de la jerarquía de las personas.

\section{Abreviaturas}

\begin{tabular}{|c|c|}
\hline$\rightarrow$ & dirección de la determinación sintáctica \\
\hline ANT & anterioridad \\
\hline ARG & caso argumentativo \\
\hline AUX & auxiliar \\
\hline CAUS & causativo \\
\hline ERG & ergativo \\
\hline FACT & factitivo \\
\hline FEM & feminino \\
\hline FOC & focalizador \\
\hline FRUST & frustrativo \\
\hline GER & gerundio \\
\hline HORT & hortativo \\
\hline
\end{tabular}




$\begin{array}{ll}\text { IMP } & \text { imperativo } \\ \text { INT } & \text { intensivo } \\ \text { LOC } & \text { locativo } \\ \text { M } & \text { masculino } \\ \text { N } & \text { marca nominal de persona } \\ \text { NARR } & \text { narrativo } \\ \text { NEG } & \text { negación } \\ \text { NOR } & \text { nominalización oracional atributiva } \\ \text { O } & \text { objeto } \\ \text { PAS.REC } & \text { pasado reciente } \\ \text { PL } & \text { plural } \\ \text { PRON } & \text { pronombre personal independiente } \\ \text { PTG } & \text { proto-tupí-guaraní } \\ \text { REL } & \text { morfema de flexión relacional } \\ \text { S } & \text { sujeto } \\ \text { SG } & \text { singular } \\ \text { VOL } & \text { volitivo }\end{array}$

\section{Referencias bibliográficas}

Anchieta SJ, Joseph de. 1595. Arte de grammatica da lingoa mais usada na costa do Brasil, Coimbra, Antonio de Mariz.

Betts, LaVera. 2012. Kagwahiva Dictionary, Anápolis - GO, SIL.

Borges, Mônica Veloso. 2006. Aspectos fonológicos e morfossintáticos da língua Avá-Canoeiro (Tupi-Guarani). Campinas: Unicamp. Tesis de doctorado. Disp. en http://www.etnolinguistica.org/tese:borges_2006

Borella, Cristina de Cássia. 2000. Aspectos morfossintáticos da língua Aweti (Tupi), Campinas: UNICAMP. Tesis de Maestría. Disponible en http://www.etnolinguistica.org/tese:borella_2000

Braga, Rafael Saint-Clair Xavier Silveira. 2016. Clitização e redobro de clíticos em Kayabí (Tupí-Guaraní, Tupí). A natureza ambígua de constituintes clíticos, Rio de Janeiro, UFRJ. Tesis de Doctorado. Disponible en http://www.etnolinguistica.org/tese:braga_2016

Cabral, Ana Suelly Arruda Câmara \& Aryon Dall'Igna Rodrigues. 2003. Dicionário da língua Asurini do Tocantins-Português, Belém / Brasília, UnB/ UFPA

Copin, François. Inédita. Grammaire du wayampi (tupi-guarani), Paris, Université de Paris 7. Tesis de Doctorado.

Coseriu, Eugenio. 1981. Lecciones de lingüística general, Madrid, Gredos.

Dietrich, Wolf. 1986. El idioma chiriguano. Gramática, textos, vocabulário, Madrid, ICI.

Dietrich, Wolf. 2000. Categorias lexicais nas línguas tupi-guarani (visão comparativa), en F. Queixal's (ed.), Des noms et des verbes en tupiguarani: état de la question, Munich, Lincom Europa: 21-37.

Dietrich, Wolf. (en prensa). Word classes and word class switching in Guaraní Syntax, en: Bruno Estigarribia et al. (eds.), Guarani Linguistics in the $21^{\text {st }}$ Century, Leiden, Brill. 
Dobson, Rose. 1997. Gramática prática com exercícios da língua Kayabi, Cuiabá, MT, SIL

Dooley, Robert A. 2006. Léxico Guarani: Dialeto Mbyá. Introdução, esboço gramatical, léxico, Cuiabá, MT, SIL.

Duarte, Fábio Bonfim. 2007. Estudos de morfossintaxe Tenetehára, Belo Horizonte, UFMG.

Gomes, Nataniel dos Santos. 2007. Clíticos, redobro e variação da ordem oracional em Kayabí (Tupí-Guaraní), Rio de Janeiro, UFRJ. Tesis de Doctorado. Disp. en http://www.etnolinguistica.org/tese:gomes_2007

González, Hebe Alicia. 2005. A grammar of Tapiete (Tupí-Guaraní), Pittsburgh, University of Pittsburgh. Tesis de Doctorado. Disponible en http://www.etnolinguistica.org/tese:gonzales_2005

Guasch, P. Antonio, S. I. 1956. El idioma guaraní. Gramática y antología de prosa y verso. Tercera edición refundida, Asunción, Casa América Moreno Hermanos.

Hoeller, P. Fray Alfredo. 1932. Grammatik der Guarayo-Sprache, Guarayos Hall (Tirol), Verlag der P.P. Franziskaner.

Jensen, Cheryl. 1998. Comparative Tupí-Guaraní Morphosyntax, en Desmond C. Derbyshire, y Geoffrey K. Pullum (eds.), Handbook of Amazonian Languages,Berlin / New York, Mouton de Gruyter: 487-618.

Julião, Maria Risoleta Silva. Inédita. Aspects morphosyntaxiques de l'anambé, Toulouse, Université de Toulouse - Le Mirail. Tesis de Doctorado

Kakumasu, James. 1986. Urubu-Kaapor, en Desmond C. Derbyshire y Geoffrey K. Pullum (eds.), Handbook of Amazonian Languages, vol. I, Berlin-New York-Amsterdam, Mouton de Gruyter: 326-403.

Lopes, Jorge Domingues 2014. Uma interface da documentação linguística e modelos lexicográficos para línguas indígenas brasileiras. Uma proposta para o Suruí-Aikewára, Brasília, UnB. Tesis de Doctorado. Disponible em http://www.etnolinguistica.org/tese:lopes_2014

Magalhães, Marina Maria Silva. 2007. Sobre a morfologia e a sintaxe da língua Guajá (Família Tupi-Guarani), Brasília, UnB. Tesis de Doctorado. Disponible en http://www.etnolinguistica.org/tese:magalhaes_2007

Meira, Sérgio y Sebastian Drude. 2013. Sobre a origem dos "prefixos relacionais" das línguas Tupí-Guaraní, Cadernos de Etnolingüística vol. 5, número 1: 1-31.

Monserrat, Ruth Maria Fonini. 2012. Prefixos pessoais em Aweti, Revista Brasileira de Linguística Antropológica 4.1: 15-28.

Monserrat, Ruth Maria Fonini y Marília Facó Soares. 1983. Hierarquia referencial em línguas Tupi, em Ensaios de Lingüística 9: 164-187.

Pereira, Antônia Alves. 2009. Estudo morfossintático do Asurini do Xingu, Campinas, UNICAMP. Tesis de Doctorado. Disponible en http://www.etnolinguistica.org/tese:pereira_2009

Praça, Walkíria Neiva. 2007. Morfossintaxe da língua Tapirapé, Brasília, UnB. Tesis de Doctorado. http://www.etnolinguistica.org/tese:praca_2007

Priest, Perry \& Anne de Priest. 1985. Diccionario Siriono y Castellano. Cochabamba (Bolivia): Instituto Lingüístico de Verano - Ministerio de Educación y Cultura. 
Ramirez, Henri y María Cristina Victorino de França. 2017. O warázu do Guaporé (tupi-guarani): primeira descrição linguística, Liames 17, 2: 7102.

Rodrigues, Aryon D. \& Wolf Dietrich. 1997. On the linguistic relationship between Mawé and Tupí-Guaraní Diachronica XIV, 2: 265-304.

Rose, Françoise. 2011. Grammaire de l'èmérillonteko, une langue tupi-guarani de Guyane Française, Leuven - Paris - Walpole, MA: Peeters.

Schermair, P. Fr. Anselmo. 1949. Gramática de la lengua sirionó, La Paz: Gráfica de A. Gamarra.

Seki, Lucy. 2000. Gramática do Kamayurá. Língua Tupi-Guarani do Alto Xingu. Campinas: Editora da Unicamp - São Paulo: Imprensa Oficial.

Souza e Silva, Auristéa Caetana. 1999. Aspectos da referência alternada em Parakanã, Belém, UFPA. Tesis de Maestría. Disponible en http://www.etnolinguistica.org/tese:silva_1999

Solano, Eliete de Jesus Bararuá. 2009. Descrição grammatical da língua Araweté, Brasília, UnB. Tesis de Doctorado. Disponible en http://www.etnolinguistica.org/tese:solano_2009

Villafañe, Lucrecia. 2004. Gramática Yuki, lengua Tupí-Guaraní de Bolivia, Tucumán, Universidad Nacional de Tucumán. 